\title{
Intranasal Oxytocin Selectively Modulates Large-Scale Brain Networks in Humans
}

\author{
Katja Brodmann,, ${ }^{1,2}$ Oliver Gruber, ${ }^{1,3}$ and Roberto Goya-Maldonado ${ }^{1,4}$
}

\begin{abstract}
A growing body of evidence indicates that the neuropeptide oxytocin (OT) alters the neural correlates of socioemotional and salience processing. Yet the effects of OT over important large-scale networks involved in these processes, such as the default mode (DM), ventral attention (VA), and cingulo-opercular (CO) networks, remain unknown. Therefore, we conducted a placebo-controlled crossover study with intranasal 24 IU OT in 38 healthy male subjects using a resting-state functional magnetic resonance imaging paradigm to investigate its impact over these three networks candidates. To understand the underlying mechanisms of the neuropeptide, we compared the intranetwork connectivity for each network candidate and also the internetwork connectivity across all networks between both treatment conditions. Based on the relevance of interindividual factors for OT effects, we correlated individual network changes with behavioral performance in a decision-making task and with impulsivity scores. Our results show that OT mainly alters connectivity in the VA, on one side reducing the coupling to regions that typically form the nodes of DM, an introspective and self-referential network, and on the other side increasing the coupling to the edges of the $\mathrm{CO}$, which is involved in salience processing. The results of the internetwork analyses confirmed the specificity of the OT effects. Indeed, we observed significant correlations with the erroneous performance during decision-making but not with the obtained impulsivity scores. Overall, our data support that the modulation of functional connectivity within the VA is a basic mechanism by which OT directs attentional resources from internal to external cues, preparing the brain for context-dependent salience processing.
\end{abstract}

Keywords: large-scale brain networks, oxytocin, functional connectivity, attention, salience, fMRI

\section{Introduction}

$\mathbf{T}$ HE IMPACT OF the neuropeptide oxytocin (OT) on the neural correlates of social functioning is not fully understood and is of significant interest. Research on the neural effects of OT in humans takes advantage of the fact that neuropeptides can be easily and non-invasive administered via nasal sprays (Born et al., 2002).

In line with animal studies, human studies have highlighted its critical role in approach-avoidance behavior (Scheele et al., 2012), pair-bonding (Walum et al., 2012), mother-pup bonding (Elmadih et al., 2014), trust (Baumgartner et al., 2008), emotion recognition (Shahrestani et al., 2013), empathy, and theory of mind (Uzefovsky et al., 2015). One of the current hypotheses about the underlying neural processes of its function is that
OT might affect brain regions that are related to social cognition (Baribeau and Anagnostou, 2015; Skuse and Gallagher, 2009). It has been found that OT receptors are distributed in brain regions associated with emotion processing, self-referential processing, and motivation, for example, the amygdala, the prefrontal cortex, and the striatum, respectively (Insel, 1992; Loup et al., 1991; Stevens et al., 2013). Increasing interest on how OT modulates networks associated with social processing has led to functional neuroimaging studies focusing on alterations in connectivity, with the amygdala as an a priori defined region of interest in resting state analyses (Kumar et al., 2015; Riem et al., 2012), and also within task-based studies (Hu et al., 2015) and in studies with clinical populations (Watanabe et al., 2015).

If OT modulates the activity and connectivity in regions linked with social and emotional processing, it would also affect

\footnotetext{
${ }^{1}$ Department of Psychiatry and Psychotherapy, Center for Translational Research in Systems Neuroscience and Psychiatry, University Medical Center, Göttingen, Germany.

${ }_{3}^{2}$ Faculdade de Medicina da Universidade de Lisboa, Instituto de Medicina Molecular, Lisbon, Portugal.

${ }^{3}$ Section for Experimental Psychopathology and Neuroimaging, Department of General Psychiatry, Heidelberg University, Heidelberg, Germany.

${ }^{4}$ The Systems Neuroscience and Imaging in Psychiatry (SNIP) Lab, Department of Psychiatry and Psychotherapy, University Medical Center, Göttingen, Germany.
}

(c) Katja Brodmann et al. 2017; Published by Mary Ann Liebert, Inc. This is an Open Access article distributed under the terms of the Creative Commons Attribution License, which permits unrestricted use, distribution, and reproduction in any medium, provided the original work is properly cited. 
the default mode network (DM) that is thought to constitute or to largely overlap with the social brain (Mars et al., 2012; Schilbach et al., 2008). The nodes of the DM have been shown to be activated when the brain is at rest and also during mind wandering or self-reflection (Buckner et al., 2008; Raichle, 2015). This large-scale network includes the medial prefrontal and anterior cingulate cortex (ACC), the precuneus, the angular gyrus, and the hippocampus, which are regions involved in several social cognitions (Raichle, 2015; Schilbach et al., 2008). The intrinsic and extrinsic functional connectivity of the DM is associated with empathy and theory of mind, emotion processing, and morality (Li et al., 2014).

These social cognitions and their underlying brain regions are also known to be modulated by OT in task-based studies (Bethlehem et al., 2013). In a neuroimaging study on decisionmaking on judgments for self and other traits (Zhao et al., 2016), OT increased other traits judgments, which was accompanied by reduced activation in the medial prefrontal cortex and its functional coupling with the precuneus and the ACC, indicating an inhibition of activity in a hub region of the DM. Some regions described as part of the DM network are frequently mentioned in studies of social cognition, although the alterations, for example, increased or decreased coupling, vary according to the sample, analysis method, and stimulation employed. During the presentation of infant laughter, functional connectivity between the amygdala and the orbitofrontal cortex, the ACC, the hippocampus, the precuneus, the supramarginal gyrus, and the middle temporal gyrus was increased by OT (Riem et al., 2012). Contrastingly, reduced connectivity between the bilateral amygdala and the right precuneus, (Kumar et al., 2015), the brainstem (Riem et al., 2013), as well as the posterior cingulate cortex, which is a central node of the DM (Li et al., 2014), was seen during restingstate functional magnetic resonance imaging (rs-fMRI).

More recently, neuroimaging studies on trauma-exposed individuals (Frijling et al., 2016) or on anxiety disorder (Gorka et al., 2015) indicate that OT might alter social salience processing, thus involving the ventral attentional (VA) and the cingulo-opercular (CO) network (Koch et al., 2014; ShamayTsoory and Abu-Akel, 2016). The VA is thought to be involved in the redirection of attention and is defined by key nodes such as the temporoparietal junction (TPJ) and the ventrolateral prefrontal cortex (vlPFC). In contrast, the $\mathrm{CO}$ is more known for top-down control and altering cognitive control and is defined by the anterior insula (AI) and the dorsal ACC (Menon, 2015; Sheffield et al., 2015; Sylvester et al. 2012; Vossel et al., 2014). Previous rs-fMRI studies have supported this assumption by determining that OT modulates the connectivity between central regions of the $\mathrm{CO}$ and the VA as, for example, the AI or the dorsal ACC and the vlPFC (Frijling et al., 2016; Koch et al., 2016). Moreover, OT increases the coupling between the amygdala and regions belonging to the salience network, such as the AI and the left inferior frontal gyrus during infant crying (Riem et al., 2011).

The influence of OT on large-scale networks and their functional relationships requires systematic investigation and relies on a sound comprehension of its modulatory effects. However, we are not aware of any rs-fMRI studies that explore whether OT modulates the functional connectivity between and within large-scale networks. We preferred a resting-state paradigm due to an interest in exploring the basic mechanisms by which OT might alter neural systems. Moreover, there is a strong equivalence between regions at rest or engaged in a task (Smith et al., 2009). Here, we investigated all independent component-driven large-scale networks potentially impacted by OT in a counterbalanced crossover placebo-controlled design.

Owing to the strong influence of OT on social behavior and its underlying neural connectivity, but also because of the latest effects seen on salience processing, we hypothesized that the DM, the $\mathrm{CO}$, and the VA would be majorly affected by OT administration. Moreover, we further investigated whether individual functional connectivity modulations were associated post hoc with behavioral performance during a decision-making task called the desire-reason-dilemma (DRD) paradigm (Diekhof and Gruber, 2010). This modified version of the DRD used socioemotional as well as neutral stimuli as attentional distracters. Thereby, we could explore whether functional connectivity changes within and between the large-scale networks are associated with reward-related processes and if this depends on socioemotional information. In addition, since OT is thought to act differently on neural correlates and behavior depending on social contextual and interindividual factors (Bartz et al., 2011; Olff et al., 2013), we also performed correlation analysis, on an exploratory basis, with nonsocial personality and impulsivity scores, as these have been shown previously to correlate with functional connectivity during the DRD (Diekhof and Gruber, 2010).

For this study, we expected (1) to see changes in functional connectivity in the main large-scale brain networks implicated in social cognition and salience processing under OT treatment and (2) that the observed changes would be associated with differences in behavioral performance during a decision-making task under OT treatment, and (3) that alterations of the networks by OT would be modulated by personal characteristics.

\section{Materials and Methods}

\section{Participants}

Forty healthy male participants from a university population were included in this double-blind, placebo-controlled, crossover study. Participants were right handed, between 18 and 35 years old, had normal or corrected-to-normal vision, and German as a first language. They were medication free and were not suffering from psychiatric, neurological, or any other diseases. In addition, they were screened for MRI contraindications and for alcohol and drug use. Participants gave written informed consent and were paid for participation. All procedures were approved by the Ethics Committee of the University Medical Center Göttingen. There is evidence that OT might act differently in females and males (Dumais and Veenema, 2016). Therefore only males were included in this study to avoid interactions e.g. between the administered OT and gonadal hormones and to keep the sample as homogenous as possible.

We were not able to calculate a power analysis beforehand for determining the sample size for the study. However, previous rs-fMRI studies exploring the effect of OT on functional connectivity between seed regions or on cerebral blood flow reported significant changes by intranasal administration of OT with the use of 16 participants per treatment condition in a between-subject design (Paloyelis et al., 2016) or by the use of 15 participants in crossover designs (Kumar et al., 2015; Sripada et al., 2012). Therefore, a sample size of $>35$ participants in a crossover design seemed to be more than sufficient to find OT-induced changes in large-scale networks. 


\section{Procedure and pharmacological intervention}

All participants underwent an rs-fMRI scan and decisionmaking paradigm in a pseudorandomized order under OT and placebo treatment, which occurred on 2 consecutive days. The day prior to the scanning session, participants filled out a battery of self-rating questionnaires, including the Temperament and Character Inventory (TCI) and Barratt Impulsiveness Scale (BIS) (Supplementary Table S1; Supplementary Data are available online at www.liebertpub.com/brain). Approximately $30 \mathrm{~min}$ before the fMRI scan, participants self-administered intranasal 24 IU OT (Syntocinon-Spray, Novartis, Basel, Switzerland) the most commonly used dose in this field of research, or placebo, according to a counterbalanced randomization order prepared by the Hospital Pharmacy of the University of Heidelberg (InPhaSol). The placebo spray was produced to look identical and have the same content as the OT spray, except for the absence of the neuropeptide.

Although there is ample evidence from animal studies, there is still limited knowledge of how much intranasal OT reaches the human brain. A pharmacological study in awake macaques by Freeman and colleagues (2016) reported increased OT levels in CSF after intranasal administration. Interestingly, the authors did not find a relationship between baseline plasma OT and CSF OT as they could not find an effect of intranasal OT on blood plasma level, suggesting a lack of correlation between peripheral and central levels. An early study demonstrated that vasopressin, a similar neuropeptide, was sustained in cerebrospinal fluid and did not reach baseline concentration within 80 min after intranasal administration in humans (Born et al., 2002). Moreover, a recent rs-fMRI study showed an altered pattern in regional blood flow in humans within a 25-78-min time measurement after OT administration (Paloyelis et al., 2016).

Based on these studies, we assume that OT was centrally present during the decision-making task and the restingstate paradigm, which took place $30 \mathrm{~min}$ and $75 \mathrm{~min}$ after administration, respectively.

\section{Tasks}

All experiments were conducted with the Presentation ${ }^{\circledR}$ software (Neurobehavioral Systems, Albany).

In the main part of the study, participants underwent a 6 min rs-fMRI paradigm. During rs-fMRI, participants were instructed to keep their eyes open, focus on a fixation cross in the middle of the screen, avoid detaining themselves on particular thoughts, and to let their minds-wander. We checked for compliance with the instructions after screening sessions and all participants confirmed.

Immediately before undergoing the resting-state scan, participants performed a modified version of the DRD. Prior to this, participants conducted a simple operant conditioning task outside the scanner in which they learned that two specific colors were immediately rewarded with 10 points (conditioned reward, CR). In the actual experiment, two colors were defined as targets in the beginning of each block and had to be selected in the following trials to gain 60 points in the end. Otherwise, the outcome was 0 . In addition, whenever a $\mathrm{CR}$ was shown, participants received bonus points by collecting them (10 points each).

Due to different combinations of colors, the participants had to adjust their selection preferences depending on the specific trial type. In the reason or target trial, the target color was paired with a no-target color and had to be selected. In the desire or reward trial, the CR was featured together with a no-target color and was free to be selected to obtain the reward (Supplementary Fig. S1). In the dilemma trial, the CR was paired with a target color and, therefore, was not free to be selected. Control trials were defined by presentation of two no-target colors. In this modified version, in each trial type, the task-relevant stimuli (colors) were presented with social stimuli (neutral, happy, and fearful faces) or with a control stimulus (gray oval). More details on the DRD task are presented in the Supplementary Data.

\section{Image acquisition and preprocessing}

MRI scans were conducted using a 3T scanner (Magnetom TRIO, Siemens Healthcare, Erlangen, Germany). Thirty-six axial slices (voxel size, $3 \times 3 \times 3 \mathrm{~mm}^{3}$; gap $=20 \%$ ) were acquired in ascending direction using a $\mathrm{T} 2 *$-sensitive echo planar imaging sequence (interscan interval, $1.9 \mathrm{~s}$; echo time, $30 \mathrm{~ms}$; flip angle, $70^{\circ}$ ). A total of 188 volumes were obtained in the course of $6 \mathrm{~min}$ resting-state session. In addition, a highresolution $\left(1 \times 1 \times 1 \mathrm{~mm}^{3}\right)$ structural image was acquired by a three-dimensional, T1-weighted, gradient-echo (MPRAGE) sequence. Preprocessing of the fMRI data was performed with SPM8 (Wellcome Trust Centre for Neuroimaging, University College London, United Kingdom; www.fil.ion.ucl.ac.uk/ spm) and included standard slice timing, realignment and unwarping, coregistration, and normalization into Montreal Neurological Institute (MNI) standard stereotactic space.

To check whether differential functional connectivity between treatment conditions might have been caused by unequal head motion, we applied the approach developed by Van Dijk and colleagues (2012) and compared the individual root mean square of frame-by-frame displacements between treatment conditions using a paired two-sample $t$-test. Next, nuisance factors were removed to produce a clearer image by regressing out the six movement parameters, white matter, and cerebrospinal fluid signals and their first temporal derivatives.

\section{Independent component analysis and comparison of networks}

The resulting images were input into a temporal concatenation of group-independent component analysis (gICA) in FSL MELODIC (Chen et al., 2008; Smith et al., 2009) to identify large-scale network components across treatment conditions by a data-driven approach. The gICA resulted in 10 components from which 5 components were identified as DM, CO, VA, visual network (VS), and fronto-parietal (FP) networks, with the remaining components defined as artifacts based on visual inspection of resulting spatiotemporal distributions. Additionally, our output of five reliable resting-state networks is in line with typical network distributions (Deco and Corbetta, 2011). Individual back transformation was performed for each component with Butterworth band-filtered $(0.01-0.1 \mathrm{~Hz})$ first Eigenvariate time-courses and followed by GLM and Fisher's r-to-z transformation.

The between-treatment condition comparison of neural correlates was performed in our a priori network candidates using a one-way ANOVA model with a statistical threshold of $p<0.001$, cluster corrected $(\mathrm{k}>13)$ for multiple comparisons, based on the Monte Carlo simulation using the AlphaSim implementation in RESting-state fMRI data analysis Toolkit 
(REST) (Song et al., 2011). Cluster-wise analyses were run on within-network connectivity of the a priori network candidates to evaluate changes of functional connectivity by OT treatment in contrast to placebo. Large-scale networks for each treatment condition, as well as the differences between them, were visualized with MRIcron (Rorden et al., 2007).

Furthermore, Pearson's correlation coefficients were calculated to identify the differences across the large-scale networks within and between the two treatment conditions and correlation matrices were produced by averaging correlations for each pair of networks over all subjects. The correlation maps were observed with MATLAB 2012a (The MathWorks, Inc., Natick, MA). To estimate treatment condition differences in the matrices, correlation coefficients were r-to-z transformed and entered into a paired two-sample $t$-test with a $p$-value Bonferroni corrected for multiple testing (five networks: $p<0.5 /$ $5=0.01)$. This internetwork analysis was done to support the results of the intranetwork analysis with SPM with reference to the issue of potential false positive results using cluster correction for multiple testing, as demonstrated in a recent fMRI method article (Eklund et al., 2016).

\section{Behavioral analysis and correlation analysis between imaging and behavioral results}

Error rates for the different task conditions (target, reward, and dilemma) were compared between both treatment conditions by applying a two-way repeated ANOVA. Furthermore, we disentangled observed significant effects with paired $t$-tests. Afterward, we calculated a correlation analysis post hoc to explore whether the OT induced changes in functional connectivity within the VA were associated with the observed differences in behavioral performance during the decision-making task. We chose only the VA for the correlation analysis as it was the only network in which the withinnetwork changes (reaching areas that are typically nodes of the DM) were confirmed by the internetwork analysis. There- fore, we created a mask of the differences in the withinnetwork changes between treatment conditions and extracted these parameter estimates. The parameter estimates were correlated only with the behavioral data, which showed a significant treatment effect in the behavioral analysis, namely error rates in target trials. To account for multiple testing correction, the $p$-value was set to $p<0.5 / 2=0.025$.

Scores of the personality and impulsivity questionnaires were included as covariates of interest in the group comparison to evaluate potential influences of individual characteristics over functional connectivity changes. A separate model was run for each subscale and total scores. The existence of a modulation was determined by changes in functional connectivity within the network candidates at a more flexible exploratory threshold of $p<0.005$. In this case, we extracted the parameter estimates with a sphere of $6 \mathrm{~mm}$ of the main analysis (to avoid circularity) and correlated it with the specific scale (Pearson's $r$ correlation, 2-tailed). We applied all four subscales and total scores of the TCI, all three subscales, and the total scores of the BIS for a sum total of nine scores. Therefore, the $p$-value was set to $p<0.5 / 9=0.006$ to correct for multiple testing. All behavioral and correlation analyses were calculated by using SPSS IBM Statistics Version 23.

\section{Results}

Two participants were excluded from the resting-state paradigm analysis and a further four participants were excluded from the behavioral correlation analysis because of movement artifacts, voluntary dropout, technical issues, and performance $<75 \%$. Therefore, a total of 38 participants (mean age in years $24.95 \pm 3.39$ ) were included in the resting-state analysis. To discard the possibility of differences in frame-by-frame displacement across treatment conditions resulting in false positive results in the functional connectivity comparisons, we compared individual root mean square of relative displacements from adjacent volumes between conditions (Materials

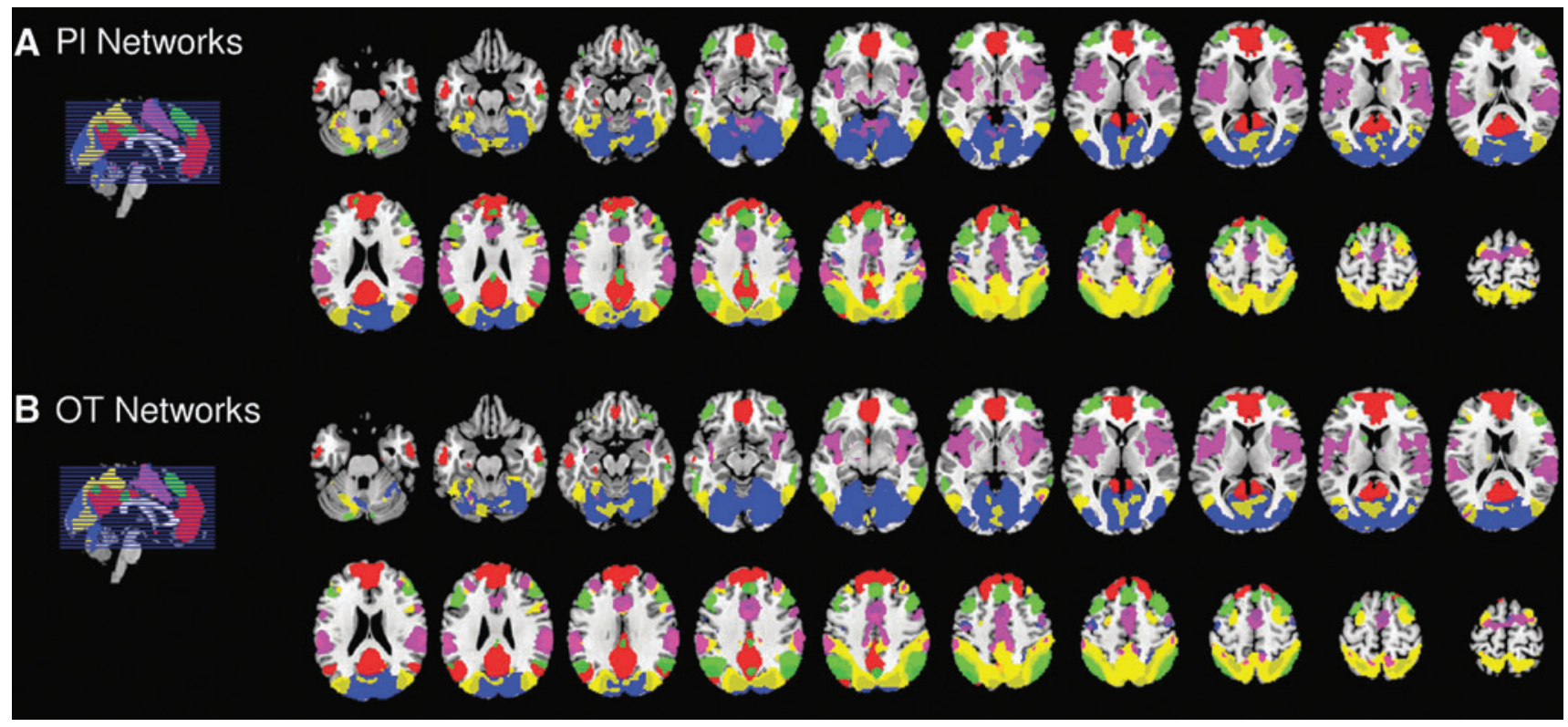

FIG. 1. Functional connectivity across networks within Pl (A) and OT (B) treatment conditions. Networks were identified after gICA: VS (blue), DM (red), VA (green), FP (yellow), and CO (pink). gICA, group-independent component analysis, Pl, placebo; OT, oxytocin; VS, visual; DM, default mode; VA, ventral attention; FP, frontoparietal; CO, cingulo-opercular. 
FIG. 2. Influence of OT on functional connectivity within VA, CO, and DM. (A) FC within VA after Pl (blue) and OT (yellow) treatment. Analysis of VA revealed that OT reduced FC (green) to nodes of the DM (brown) and increased FC (red) to edges of $\mathrm{CO}$ (brown). (B) FC within $\mathrm{CO}$ after Pl (blue) and OT (yellow) treatment. Analysis of $\mathrm{CO}$ (brown) revealed that OT increased FC (red) to the right NAcc and reduced $\mathrm{FC}$ (blue) to the VTA. (C) FC within DM after Pl (blue) and OT (yellow) treatment. There were no statistical significant differences after treatment in FC within this network. For observation purposes, thresholded SPM maps of both treatment conditions and networks were overlaid on MRIcron templates (AlphaSim correction at $p<0.001$ with $\mathrm{k}<13$ for treatment condition comparisons). Color bars represent the intensity of $t$-values. FC, functional connectivity.

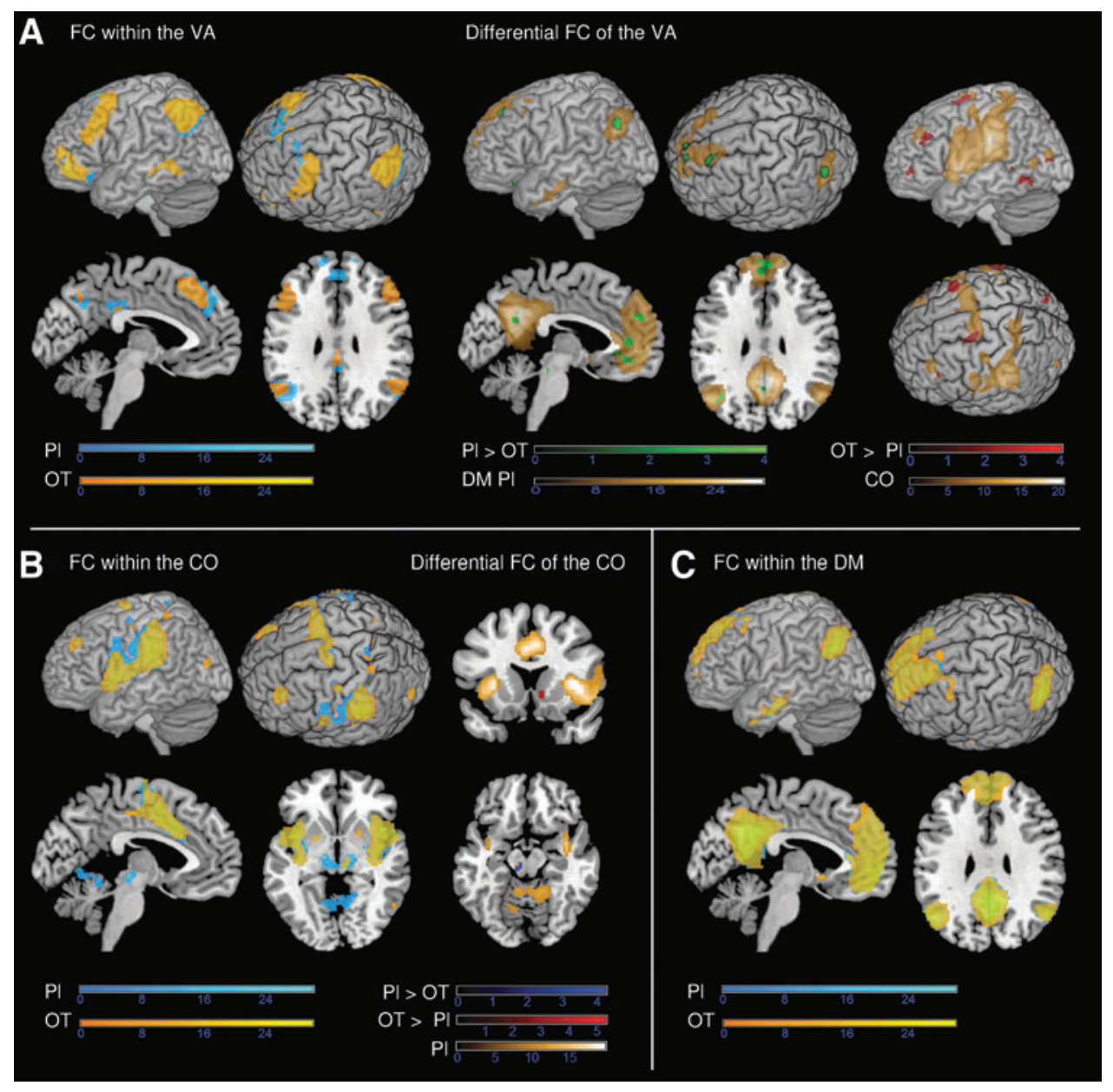

and Methods section). There were no significant differences (in $\mathrm{mm}$, placebo: $\mathrm{M}=1.29 \mathrm{SD}=0.95$; OT: $\mathrm{M}=1.34 \mathrm{SD}=0.93$; $\mathrm{T}=0.24, p=0.810$ ), so we could exclude this possibility.

The gICA yielded five large-scale networks, namely the $\mathrm{VS}$, the VA, the DM, the FP, and the CO, defined according to the spatiotemporal configuration presented in multiple studies (for instance, Deco and Corbetta, 2011; Sylvester et al., 2012; Vaidya and Gordon, 2013). All networks were well represented in both treatment conditions, as shown in Figure 1 and in more detail in Supplementary Figures S2 and S3

\section{Intranetwork functional connectivity}

At first we identified within-network differences between treatment conditions (Supplementary Table S2). The strongest effect of OT treatment concerned functional connectivity within the VA (Fig. 2A). Within the VA, OT in contrast to placebo significantly reduced functional connectivity to regions typically belonging to the nodes of the DM, such as medial frontal, pregenual, and subgenual ACC regions along with the precuneus, posterior inferior parietal lobe, and hippocampus (Fig. 2A, green blobs). In contrast, OT significantly increased functional connectivity to regions corresponding to the edges of the $\mathrm{CO}$, such as the superior AI, postcentral and superior parietal regions, medial septum, and inferior parietal lobe (Fig. 2A, red blobs).

In addition, OT decreased functional connectivity within the ventral tegmental area (VTA), the central operculum, the infe- rior AI, and the brain stem (Supplementary Table S2). The analysis of the $\mathrm{CO}$ also revealed reduced functional connectivity in the VTA and cerebellum with the OT versus placebo treatment (Fig. 2B and Supplementary Table S2). Conversely, OT strengthened functional connectivity within this network in the nucleus accumbens (NAcc) and supramarginal gyrus (Fig. 2B and Supplementary Table S2). Lastly, the analysis of the DM showed that within this network no direct influence of OT in contrast to placebo was statistically relevant (Fig. 2C).

\section{Internetwork functional connectivity}

Finally, internetwork functional connectivity in both treatment conditions is shown for all networks with correlation matrices (Fig. 3). OT significantly decreased functional connectivity between the VA and the DM $(t=3.57, p<0.001)$. There was also a trend-level decrease of functional connectivity between the FP and the DM, which did not survive correction for multiple testing $(t=1.98, p=0.055)$. Moreover, OT slightly increased functional connectivity between the VA and the FP as well as between the FP and the $\mathrm{CO}$, but these findings were not significant (both $p>0.05$ ).

\section{Behavioral data and correlation between functional connectivity changes and behavioral data}

OT led to an increase in the error rate during the DRD for reason or target trials (Fig. 4). The ANOVA on error rate 


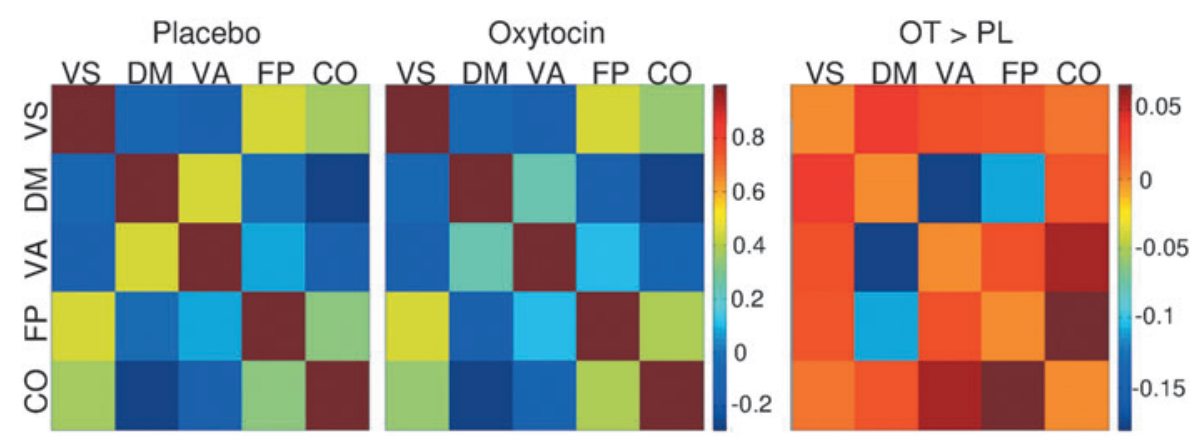

FIG. 3. Internetwork functional connectivity matrices for Pl and OT treatment conditions. Pairwise Pearson's correlations between time courses of networks are displayed for each treatment condition. Change in network connectivity after OT treatment is displayed in an additional correlation matrix. Only the difference in FC between VA and DM was significant $(p<0.01$ Bonferroni corrected for multiple testing). The reduced connectivity between FP and DM was at a trend level $(p=0.055)$. Colors of the matrices represent the intensity of the averaged z-scores.

with the factors treatment (OT/P1) and trial type (target, desire/bonus, and dilemma) revealed a main effect of treatment $(\mathrm{F}=5.47 ; p<0.05)$, trial type $(F=5.69 ; p<0.005)$, and an interaction effect between treatment and trial type $(F=4$; $p<0.05)$. Post hoc $t$-tests uncovered that under OT more errors were made in the target trials, the trials in which a target color was paired with a no-target color, in comparison with placebo $(t=6.94, p<0.0001)$. Therefore, the correlation between the percentage of errors and estimates of VA is presented for target trials.

The subsequent correlation analysis with the parameter estimates of the VA for each treatment condition showed that the weaker the functional connectivity reaching typical node regions of the DM (Fig. 2A, green blobs) the higher was the error rate for the OT condition $(r=-0.436, p=0.010)$ (Fig. 5, top). The placebo condition did not show such a correlation ( $r=-0.210, p>0.025)$ (Fig. 5, bottom).

Here, the question arose whether OT would influence performance differently when stimuli with socioemotional or nonsocial content were presented (emotional vs. scrambled faces). Therefore, we calculated an ANOVA on the factors treatment (placebo/OT) and social content (yes/no). There was a main effect of treatment $(F=37.01 ; p<0.0001)$, but no effect of social content and no interaction effect (all $p>0.05)$. Post hoc tests revealed that under OT, participants performed worse with nonsocial $(t=4.47 ; p<0.0001)$ and social content $(t=5.95 ; p<0.0001)$ in comparison with the placebo condition.

To investigate whether nonsocial interindividual factors have an influence on the effect of OT over the candidate networks, we correlated the TCI and BIS scales with significant changes in the functional connectivity of the VA under OT treatment. However, we did not find any correlation between these personality traits and functional connectivity under OT treatment that survived correction for multiple comparisons.

\section{Discussion}

To the best of our knowledge, we have conducted the first rs-fMRI study to systematically investigate the influence of exogenous OT over the most representative large-scale brain networks for social cognition and salience processing, namely the DM, CO, and VA networks. Our findings show that OT influenced the VA by decreasing cross-talk to regions typically considered to be DM nodes, and strengthened functional connectivity to the edges of the CO. In addition, OT directly impacted functional connectivity within the $\mathrm{CO}$, but not functional connectivity within the DM. Moreover, functional

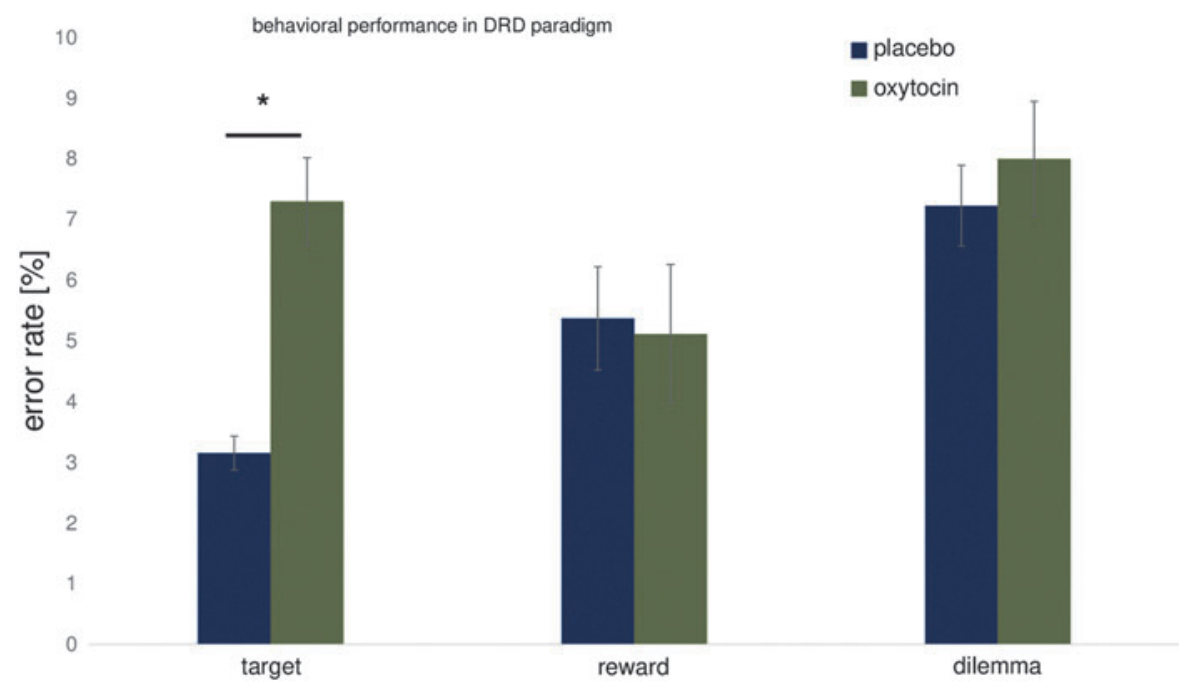

FIG. 4. Error rate for different trial types during the DRD. Under OT treatment, the error rate was increased for target trials $(p<0.05)$ but not for reward or dilemma trials in comparison with placebo treatment (all $p>0.05)$. DRD, desirereason-dilemma. $* p<0.0001$, $t$-test. 

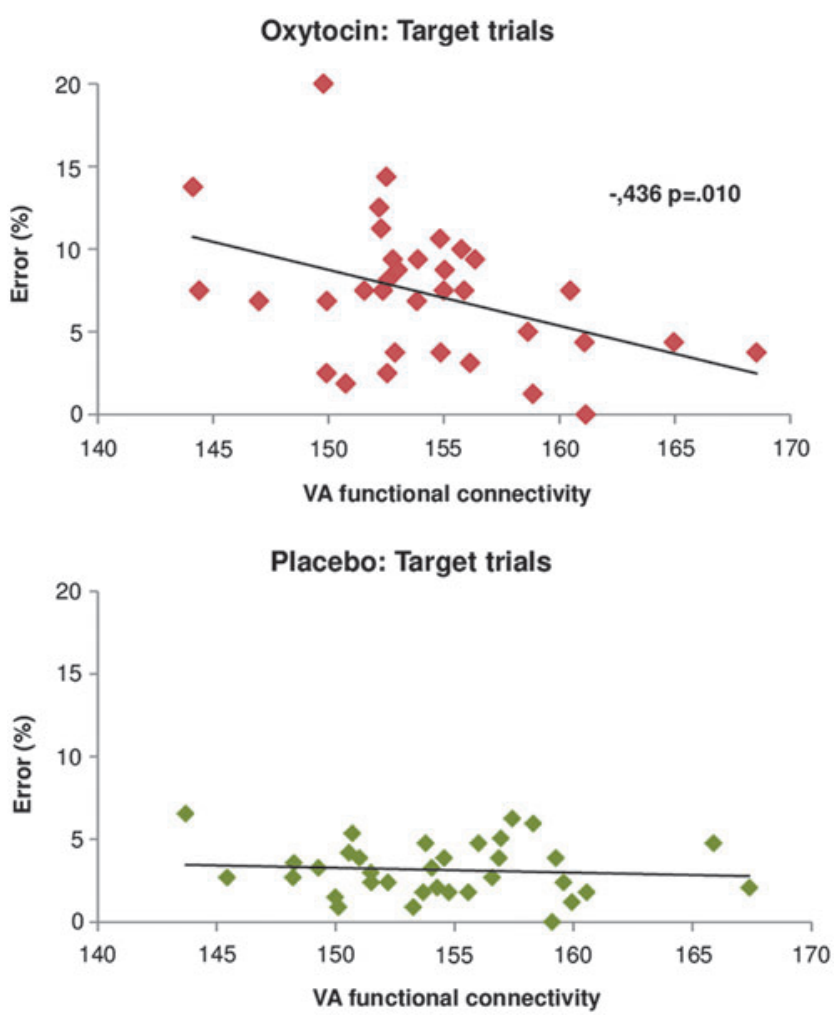

FIG. 5. Correlation analysis between VA and error rate during the DRD for OT and placebo treatment conditions. Functional connectivity of the VA correlated negatively with the error rates in target trials for the OT treatment condition $(r=-0.436 ; p=0.01)$ but not for the placebo treatment condition $(p>0.05)$.

connectivity within the VA was negatively correlated with the error rate in a decision-making paradigm under OT treatment but not under placebo treatment. This study sheds new light on the network mechanisms by which OT might regulate the salience of cues and influence attentional processes.

Based on our data, OT seems to acutely modulate the intersection of DM and CO networks, with the VA as the direct target. The VA has been called a "circuit breaker," as it interrupts the top-down-directed attention from the FP (Vossel et al., 2014), allowing the switch to reorient attentional resources to salient stimuli present in the external environment with the CO (Corbetta and Shulman, 2002; Corbetta et al., 2008) and vice versa. Building on this evidence, our results show that the VA under OT has reduced access to break the DM circuit, but also increased access to potentiate attention reallocation to the $\mathrm{CO}$ with an increased capacity of external salience processing.

Unbalanced cross-talk between these three networks is in line with the social salience hypothesis of OT, where an increased sensitivity to external contextual social cues (e.g., competitive vs. cooperative) in the environment can occur (Olff et al., 2013; Shamay-Tsoory and Abu-Akel, 2016). Further evidence for this assumption arises from other neuroimaging studies that display increased attentional effects relevant emotional or social stimuli after OT use. For example, in one behavioral study the authors could observe that OT increased the attention toward both positive and negative facial expressions in healthy male test subjects (Tollenaar et al., 2013), whereas another study conducted on chronically depressed patients found that attention was selectively redirected to happy faces and decreased toward angry facial expressions by the use of OT (Domes et al., 2016). In addition, a neuroimaging study focusing on the effects of OT on neural activity in the amygdala could not only show that OT selectively modulated the activation of different amygdala subregions depending on the valence of the presented emotional facial expressions, but also that OT-induced gaze shifts toward the eye region of a face irrespective of the presented emotional expression (Gamer et al., 2010).

Despite the differences regarding OT modulation of attention depending on the tested species, the presentation of specific emotion and whether the human sample was healthy or not, the results reveal that OT essentially modulates attentional resources. One major hub for these switches and the reallocation of attention within the VA could be the TPJ, a region containing parts of the superior temporal sulcus, the angular gyrus, and the inferior parietal lobe, which has also been found to be affected by OT in a task-based study ( $\mathrm{Hu}$ et al., 2016). The TPJ is assumed to be involved in perspective taking and is, therefore, implicated in switching between networks (Corbetta et al., 2008).

However, there is evidence against the exclusive role of the TPJ in the reorientation of networks (DiQuattro et al., 2014) and some authors assume that the switch rather relies on the insular cortex, which is also activated during tasks requiring reorientation and switching between networks (Sridharan et al., 2008). Under OT treatment, we found both a modulation of the superior temporal sulcus/angular gyrus and the AI, indicating that OT might support the functional decoupling between internal processing and external cues, redirecting the attention, and switch between the large-scale networks.

According to the social salience theory (Shamay-Tsoory and Abu-Akel, 2016), OT regulates mainly the salience network and through this modulation also the reorientation of attention to external cues might occur. The AI, as a part of the VA or CO, is not only implicated in network switching but seems to also have a prominent role in salience processing (Menon, 2015; Menon and Uddin, 2010). A meta-analysis demonstrated that OT consistently enhanced activation in the AI (Wigton et al., 2015). Even though the authors interpreted this finding as a sign for increased emotion processing, a modulation of the salience network or attention by OT is just as feasible. Moreover, we found alterations of connectivity within the $\mathrm{CO}$ to other major hubs associated with salience and motivational behavior, such as the VTA and the ventral striatum, including the NAcc (Menon, 2015). These structures are fundamentally involved in the dopaminergic reward system (Haber and Knutson, 2010), reinforcing in this context the idea of an interaction between salience processing and reward.

The effects of OT on neural activity in VTA and NAcc have been shown in human studies using monetary and social reward learning tasks ( $\mathrm{Hu}$ et al., 2015) and in nonhuman studies by an interaction between the neuropeptide and its receptor with the dopaminergic reward system (Shahrokh et al., 2010; Young et al., 2014). Therefore, the significant attenuation of the coupling with the VTA and the increased connectivity to the NAcc within the CO shown in our results might also reflect a regulation mechanism of the reward and salience processing systems by OT. 
Behavioral data and its correlation with the functional connectivity of the VA might more directly assess the effects of OT on attentional processing. Surprisingly, we identified no behavioral effect of OT treatment for trials including a bonus stimulus, which generally increases bottom-up-related activation in the reward/salience system (Diekhof and Gruber, 2010). Moreover, there was also no difference in the performance between the task-irrelevant socioemotional and nonsocial stimuli. OT decreased behavioral performance in target trials independent of the social or neutral content of the stimuli. Indeed, the decrease in functional connectivity within the VA was negatively correlated with error rate in target trials in the OT but not in the placebo condition.

Whereas the selection of targets relies mostly on top-down or goal-driven attention, also termed endogenous attention, the distraction by the task-irrelevant stimuli switches attention to external and salient stimuli, a process which is termed exogenous attention (Carretié, 2014). Furthermore, the neural correlates of exogenous attention are assumed to be in the vental attention system that is covered by the VA (Carretié, 2014; Chica et al., 2013). This could explain our results based on a single use of OT. One of the key players in exogenous attention processing might be the TPJ, which was associated with the orienting of exogenous but not endogenous attention in a transcranial magnetic stimulation study on spatial attention (Chica et al., 2011). Regarding the correlation found in our study between the VA and the error rate in target trials, OT might have mediated increased salience of distractors by changing endogenous attention from the selection of targets to task-irrelevant (social and nonsocial) distractors. The reason why the change only occurred during the target trials and not during the other task conditions should be investigated in further studies. Possible causes might rely on differences in the task difficulty between the trial types (Siciliano et al., 2017) or on the additional involvement of the reward system by a bonus stimulus (Wei et al., 2016).

We could not show that nonsocial interindividual factors (Bartz et al., 2011; Olff et al., 2013) such as impulsivity traits, were associated with differential modulation of network connectivity after OT treatment. Perhaps such associations are rather observed in task-based studies, wherein neural regions are recruited and that are known to be correlated with impulsivity traits.

The limitations of using an rs-fMRI approach must be considered. First, we cannot determine whether the modulation of networks in this study implies a subsequent modification of behavior. Although we include post hoc a task-based paradigm for correlation analysis, we cannot draw direct conclusions regarding the regional activations and behavioral alterations driven by social and salience processing. Moreover, connectivity results cannot be generally translated to the activation findings in relevant regions (e.g., AI or VTA) seen in other studies. Therefore, we also cannot interpret the difference in connectivity to the NAcc and the VTA within the $\mathrm{CO}$. Furthermore, the resting-state paradigm was conducted late in the proposed window of pharmacological actions of OT (Paloyelis et al., 2016). It might be the case that the level of OT in the brain was already decreasing and, therefore, we would have seen stronger changes in the large-scale networks (including the DM) at an earlier time.

Although the mechanisms of how OT influences behavior and its underlying neural activity in healthy subjects are still not clear, this neuropeptide has already been used in various experimental and clinical trials in autism (Watanabe et al., 2015), schizophrenia (Shin et al., 2015), and post-traumatic stress disorder (PTSD) (Koch et al., 2016). Considering the development of exploring new possible biomarkers for psychiatric disorders using large-scale networks in patients (Goya-Maldonado et al., 2015; Sheffield et al., 2015; Sylvester et al., 2012), future rs-fMRI studies should focus to a greater extent on the modulation of large-scale networks by OT in patient groups. Exploring the effects of OT on large-scale networks could shed new light on the specific and selective action of the neuropeptide and might promote optimized pharmacological therapies based on specific dysfunctional networks. For example, disorders such as PTSD, which is associated with deficits in salience processing and in reorientation of attention, might benefit from these new results. Furthermore, understanding the resting-state effects of OT might lead to better evaluation of clinical responses to OT administration.

\section{Conclusion}

To conclude, our study contributes to a better understanding of the effects of the neuropeptide OT in modulating the relationship between networks responsible for attentional (VA), emotional (DM), and salience (CO) processing. These results are in line with the social salience hypothesis, which proposes a framework of OT increasing sensitivity to contextdependent social cues and, therefore, shifting resources toward social affiliations. Therefore, we conclude that OT might prepare the individual to perceive and engage with external information that demands attention and adaptive responses, in situations such as social interactions.

\section{Acknowledgments}

We thank the Department of Psychiatry and Psychotherapy of the University Medical Center Göttingen in supporting this work and we thank the local MRI staff for their valuable contributions to this study. Moreover, we thank Tracy Erwin-Grabner and Grant Sutcliffe for proof reading the manuscript. Roberto Goya-Maldonado was supported by the Federal Ministry of Education and Research (Bundesministerium für Bildung und Forschung, BMBF: 01 ZX 1507, "PreNeSt - e:Med").

\section{Author Disclosure Statement}

All authors reported that no competing financial interests exist.

\section{References}

Baribeau DA, Anagnostou E. 2015. Oxytocin and vasopressin: linking pituitaryneuropeptides and their receptors to social neurocircuits. Front Neurosci 9:335.

Bartz JA, Zaki J, Bolger N, Ochsner KN. 2011. Social effects of oxytocin in humans: context and person matter. Trends Cogn Sci 15:301-309.

Baumgartner T, Heinrichs M, Vonlanthen A, Fischbacher U, Fehr E. 2008. Oxytocin shapes the neural circuitry of trust and trust adaptation in humans. Neuron 58:639-650.

Bethlehem RAI, van Honk J, Auyeung B, Baron-Cohen S. 2013. Oxytocin, brain physiology, and functional connectivity: A review of intranasal oxytocin fMRI studies. Psychoneuroendocrinology 38:962-974. 
Born J, Lange T, Kern W, McGregor GP, Bickel U, Fehm Hl. 2002. Sniffing Neuropeptides: a transnasal approach to the human brain. Nat Neurosci 5:514-516.

Buckner RL, Andrews-Hanna JR, Schacter DL. 2008. The Brain's Default Network: Anatomy, Function, and Relevance to Disease. Ann N Y Acad Sci 1124:1-38.

Carretié L. 2014. Exogenous (automatic) attention to emotional stimuli: a review. Cogn Affect Behav Neurosci 14:12281258.

Chen S, Ross TJ, Zhan W, Myers CS, Chuang K-S, Heishman SJ, et al. 2008. Group independent component analysis reveals consistent resting-state networks across multiple sessions. Brain Res 1239:141-151.

Chica AB, Bartolomeo P, Valero-Cabré A. 2011. Dorsal and ventral parietal contributions to spatial orienting in the human brain. J Neurosci 31:8143-8149.

Chica AB, Bartolomeo P, Lupiánez J. 2013. Two cognitive and neural systems for endogenous and exogenous spatial attention. Behav Brain Res 237:107-123.

Corbetta M, Shulman GL. 2002. Control of goal-directed and stimulus-driven attention in the brain. Nat Rev Neurosci 3:215-229.

Corbetta M, Patel G, Shulman GL. 2008. The reorienting system of the human brain: from environment to theory of mind. Neuron 58:306-324.

Deco G, Corbetta M. 2011. The dynamical balance of the brain at rest. Neuroscientist 17:107-123.

Diekhof EK, Gruber O. 2010. When desire collides with reason: functional interactions between anteroventral prefrontal cortex and nucleus accumbens underlie the human ability to resist impulsive desires. J Neurosci 30:1488-1493.

DiQuattro NE, Sawaki R, Geng JJ. 2014. Effective connectivity during feature-based attentional capture: evidence against the attentional reorienting hypothesis of TPJ. Cereb Cortex 24: 3131-3141.

Domes G, Normann C, Heinrichs M. 2016. The effect of oxytocin on attention to angry and happy faces in chronic depression. BMC Psychiatry 16:92.

Dumais KM, Veenema AH. 2016. Vasopressin and oxytocin receptor systems in the brain: Sex differences and sex-specific regulation of social behavior. Front Neuroendocrinol 40: $1-23$.

Eklund A, Nichols TE, Knutsson H. 2016. Cluster failure: why fMRI inferences for spatial extent have inflated false-positive rates. Proc Natl Acad Sci U S A 113:7900-7905.

Elmadih A, Wai Wan M, Numan M, Elliott R, Downey D, Abel KM. 2014. Does oxytocin modulate variation in maternal caregiving in healthy new mothers? Brain Res 1580:143150.

Frijling JL, van Zuiden M, Koch SBJ, Nawijn L, Veltman DJ, Olff M. 2016. Intranasal oxytocin affects amygdala functional connectivity after trauma script-driven imagery in distressed recently trauma-exposed individuals. Neuropsychopharmacology 41:1286-1296.

Gamer M, Zurowski B, Buchel C. 2010. Different amygdala subregions mediate valence-related and attentional effects of oxytocin in humans. Proc Natl Acad Sci USA 107:9400 9405.

Gorka SM, Fitzgerald DA, Labuschagne I, Hosanagar A, Wood AG, Nathan PJ, et al. 2015. Oxytocin modulation of amygdala functional connectivity to fearful faces in generalized social anxiety disorder. Neuropsychopharmacology 40:278-286.

Goya-Maldonado R, Weber K, Trost S, Diekhof E, Keil M, Dechent P, et al. 2015. Dissociating pathomechanisms of de- pression with fMRI: bottom-up or top-down dysfunctions of the reward system. Eur Arch Psychiatry Clin Neurosci 265: $57-66$.

Haber SN, Knutson B. 2010. The reward circuit: linking primate anatomy and human imaging. Neuropsychopharmacology 35:4-26.

Hu J, Qi S, Becker B, Luo L, Gao S, Gong Q, et al. 2015. Oxytocin selectively facilitates learning with social feedback and increases activity and functional connectivity in emotional memory and reward processing regions: oxytocin facilitates social feedback learning. Hum Brain Mapp 36:2132-2146.

Hu Y, Scheele D, Becker B, Voos G, David B, Hurlemann R, et al. 2016. The effect of oxytocin on third-party altruistic decisions in unfair situations: an fMRI Study. Sci Rep 6:20236.

Insel TR. 1992. Oxytocin-a neuropeptide for affiliation: evidence from behavioral, receptor autoradiographic, and comparative studies. Psychoneuroendocrinology 17:3-35.

Koch SBJ, van Zuiden M, Nawijn L, Frijling JL, Veltman DJ, Olff M. 2014. Intranasal oxytocin as strategy for medicationenhanced psychotherapy of PTSD: salience processing and fear inhibition processes. Psychoneuroendocrinology 40: 242-256.

Koch SBJ, van Zuiden M, Nawijn L, Frijling JL, Veltman DJ, Olff M. 2016. Intranasal oxytocin normalizes amygdala functional connectivity in posttraumatic stress disorder. Neuropsychopharmacology 41:2014-2051.

Kumar J, Vollm B, Palaniyappan L. 2015. Oxytocin affects the connectivity of the precuneus and the amygdala: a randomized, double-blinded, placebo-controlled neuroimaging trial. Int J Neuropsychopharmacol 18:pii: pyu051-pyu051.

Li W, Mai X, Liu C. 2014. The default mode network and social understanding of others: what do brain connectivity studies tell us. Front Hum Neurosci 8:74.

Loup F, Tribollet E, Dubois-Dauphin M, Dreifuss JJ. 1991. Localization of high-affinity binding sites for oxytocin and vasopressin in the human brain. An autoradiographic study. Brain Res 555:220-232.

Mars RB, Neubert F-X, Noonan MP, Sallet J, Toni I, Rushworth MFS. 2012. On the relationship between the 'default mode network' and the 'social brain'. Front Hum Neurosci 6:189.

Menon V, Uddin LQ. 2010. Saliency, switching, attention and control: a network model of insula function. Brain Struct Funct 214:655-667.

Menon V. 2015. Salience Network. In: Toga AW (ed.) Brain Mapping: An Encyclopedic Reference, vol. 2. London UK: Academic Press, Elsevier, pp. 597-611.

Olff M, Frijling JL, Kubzansky LD, Bradley B, Ellenbogen MA, Cardoso C, et al. 2013. The role of oxytocin in social bonding, stress regulation and mental health: an update on the moderating effects of context and interindividual differences. Psychoneuroendocrinology 38:1883-1894.

Paloyelis Y, Doyle OM, Zelaya FO, Maltezos S, Williams SC, Fotopoulou A, Howard MA. 2016. A spatiotemporal profile of in vivo cerebral blood flow changes following intranasal oxytocin in humans. Biol Psychiatry 79:693-705.

Raichle ME. 2015. The Brain's Default Mode Network. Annu Rev Neurosci 38:433-447.

Riem MM, Bakermans-Kranenburg MJ, Pieper S, Tops M, Boksem MA, Vermeiren RR et al. 2011. Oxytocin modulates amzgdala, insula, andinferior frontal gyrus responses to infant crying: a randomiyed controlled trial. Biol Psychiatry 70:291-297.

Riem MM, van IJzendoorn MH, Tops M, Boksem MA, Rombouts SA, Bakermans-Kranenburg MJ. 2012. No laughing 
matter: intranasal oxytocin administration changes functional brain connectivity during exposure to infant laughter. Neuropsychopharmacology 37:1257-1266.

Riem MM, van IJzendoorn MH, Tops M, Boksem MA, Rombouts SA, Bakermans-Kranenburg MJ. 2013. Oxytocin effects on complex brain networks are moderated by experiences of maternal love withdrawal. Eur Neuropsychopharmacol 23: $1288-1295$.

Rorden C, Karnath HO, Bonilha L. 2007. Improving lesionsymptom mapping. J Cogn Neurosci 19:1081-1088.

Scheele D, Striepens N, Gunturkun O, Deutschlander S, Maier W, Kendrick KM, et al. 2012. Oxytocin modulates social distance between males and females. J Neurosci 32:1607416079.

Schilbach L, Eickhoff SB, Rotarska-Jagiela A, Fink GR, Vogeley K. 2008. Minds at rest? Social cognition as the default mode of cognizing and its putative relationship to the 'default system' of the brain. Conscious Cogn 17:457-467.

Shahrestani S, Kemp AH, Guastella AJ. 2013. The impact of a single administration of intranasal oxytocin on the recognition of basic emotions in humans: a meta-analysis. Neuropsychopharmacology 38:1929-1936.

Shamay-Tsoory SG, Abu-Akel A. 2016. The social salience hypothesis of oxytocin. Biol Psychiatry 79:194-202.

Sheffield JM, Repovs G, Harms MP, Carter CS, Gold JM, MacDonald III AW, et al. 2015. Fronto-parietal and cinguloopercular network integrity and cognition in health and schizophrenia. Neuropsychologia 73:82-93.

Shin NY, Park HY, Jung WH, Park JW, Yun J-Y, Jang JH, et al. 2015. Effects of oxytocin on neural response to facial expressions in patients with schizophrenia. Neuropsychopharmacology 40:1919-1927.

Siciliano RE, Madden DJ, Tallman CW, Boylan MA, Kirste J, Monge ZA, et al. 2017. Task difficulty modulates brain activation in the emotional oddball task. Brain Res 1664:74-86.

Skuse DH, Gallagher L. 2009. Dopaminergic-neuropeptide interactions in the social brain. Trends Cogn Sci 13:27-35.

Smith SM, Fox PT, Miller KL, Glahn DC, Fox PM, Mackay CE, et al. 2009. Correspondence of the brain's functional architecture during activation and rest. Proc Natl Acad Sci USA 106:13040-13045.

Song X-W, Dong Z-Y, Long X-Y, Li S-F, Zuo X-N, Zhu C-Z, et al. 2011. REST: A Toolkit for Resting-State Functional Magnetic Resonance Imaging Data Processing. PLoS One 6:e25031.

Sridharan D, Levitin DJ, Menon V. 2008. A critical role for the right fronto-insular cortex in switching between centralexecutive and default-mode networks. Proc Natl Acad Sci USA 105:12569-12574.

Sripada CS, Phan KL, Labuschagne I, Welsh R, Nathan PJ, Wood AG. 2013. Oxytocin enhances resting-state connectivity between amygdala and medial frontal cortex. Int J Neuropsychopharmacol 16:255-260.

Stevens FL, Wiesman O, Feldman R, Hurley RA, Taber KH. 2013. Oxytocin and behavior: evidence for effects in the brain. J Neuropsychiatry Clin Neurosci 25:96-102.
Sylvester CM, Corbetta M, Raichle ME, Rodebaugh TL, Schlaggar BL, Sheline YI, et al. 2012. Functional network dysfunction in anxiety and anxiety disorders. Trends Neurosci 35: 527-535.

Tollenaar MS, Chatzimanoli M, van der Wee NJA, Putman P. 2013. Enhanced orienting of attention in response to emotional gaze cues after oxytocin administration in healthy young men. Psychoneuroendocrinology 38:1797-1802.

Uzefovsky F, Shalev I, Israel S, Edelman S, Raz Y, Mankuta D, et al. 2015. Oxytocin receptor and vasopressin receptor 1a genes are respectively associated with emotional and cognitive empathy. Horm Behav 67:60-65.

Vaidya CJ, Gordon EM. 2013. Phenotypic variability in restingstate functional connectivity: current status. Brain Connect 3:99-120.

Van Dijk KRA, Sabuncu MR, Buckner RL. 2012. The influence of head motion on intrinsic functional connectivity MRI. NeuroImage 59:431-438.

Vossel S, Geng JJ, Fink GR. 2014. Dorsal and ventral attention systems distinct neural circuits but collaborative roles. Neuroscientist 20:150-159.

Walum H, Lichtenstein P, Neiderhiser JM, Reiss D, Ganiban JM, Spotts EL, et al. 2012. Variation in the Oxytocin Receptor Gene Is Associated with Pair-Bonding and Social Behavior. Biol Psychiatry 71:419-426.

Watanabe T, Kuroda M, Kuwabara H, Aoki Y, Iwashiro N, Tatsunobu N, et al. 2015. Clinical and neural effects of six-week administration of oxytocin on core symptoms of autism. Brain 138:3400-3412.

Wei P, Wand D, Ji L. 2016. Reward expectation regulates brain responses to task-relevant and task-irrelevant emotional words: ERP evidence. Soc Cogn Affect Neurosci 11:191203.

Wigton R, Radua J, Allen P, Averbeck B, Meyer-Lindenberg A, McGuire P, et al. 2015. Neurophysiological effects of acute oxytocin administration: systematic review and metaanalysis of placebo-controlled imaging studies. J Psychiatry Neurosci 40:E1-E22.

Young KA, Liu Y, Gobrogge KL, Wang H, Wang Z. 2014. Oxytocin Reverses Amphetamine-Induced Deficits in Social Bonding: Evidence for an Interaction with Nucleus Accumbens Dopamine. J Neurosci 34:8499-8506.

Zhao W, Yao S, Li Q, Geng Y, Ma X, Luo L, et al. 2016. Oxytocin blurs the self-other distinction during trait judgements and reduces medial prefrontal cortex responses. Hum Brain Mapp 37:2512-2527.

Address correspondence to: Katja Brodmann

Faculdade de Medicina da Universidade de Lisboa Instituto de Medicina Molecular Av. Professor Egas Moniz Lisbon 16490-028 Portugal

E-mail: katja.brodmann@medicina.ulisboa.pt 